\title{
Statistical Tests for Extreme Precipitation Volumes
}

\author{
Victor Korolev 1,2,3, Andrey Gorshenin $1,2, * \mathbb{C}$ and Konstatin Belyaev ${ }^{1,4}$ \\ 1 Faculty of Computational Mathematics and Cybernetics, Lomonosov Moscow State University, \\ Moscow 119991, Russia \\ 2 Federal Research Center "Computer Science and Control" of the Russian Academy of Sciences, \\ Moscow 119333, Russia \\ 3 Hangzhou Dianzi University, Hangzhou 310018, China \\ 4 P. P. Shirshov Institute of Oceanology of the Russian Academy of Sciences, Moscow 117997, Russia \\ * Correspondence: agorshenin@frccsc.ru
}

Received: 24 June 2019; Accepted: 17 July 2019; Published: 19 July 2019

\begin{abstract}
The analysis of the real observations of precipitation based on the novel statistical approach using the negative binomial distribution as a model for describing the random duration of a wet period is considered and discussed. The study shows that this distribution fits very well to the real observations and generalized standard methods used in meteorology to detect an extreme volume of precipitation. It also provides a theoretical base for the determination of asymptotic approximations to the distributions of the maximum daily precipitation volume within a wet period, as well as the total precipitation volume over a wet period. The paper demonstrates that the relation of the unique precipitation volume, having the gamma distribution, divided by the total precipitation volume taken over the wet period is given by the Snedecor-Fisher or beta distributions. It allows us to construct statistical tests to determine the extreme precipitations. Within this approach, it is possible to introduce the notions of relatively and absolutely extreme precipitation volumes. An alternative method to determine an extreme daily precipitation volume based on a certain quantile of the tempered Snedecor-Fisher distribution is also suggested. The results of the application of these methods to real data are presented.
\end{abstract}

Keywords: wet periods; total precipitation volume; asymptotic approximation; extreme order statistics; random sample size; testing statistical hypotheses

MSC: 62G30; 62E20; 62P12; 65C20

\section{Introduction}

Estimates of regularities and trends in heavy and extreme daily precipitation are important for understanding climate variability and change at relatively small or medium time horizons [1-3]. However, such estimates are much more uncertain compared to those derived for mean precipitation or total precipitation during a wet period [4]. This uncertainty is due to the fact that, first, estimates of heavy precipitation depend closely on the accuracy of the daily records; they are more sensitive to missing values. Second, uncertainties in the estimates of heavy and extreme precipitation are caused by the inadequacy of the mathematical models used for the corresponding calculations. Third, these uncertainties are boosted by the lack of reasonable means for the unambiguous (algorithmic) determination of extreme or anomalously heavy precipitation amplified by some statistical significance problems owing to the low occurrence of such events. As a consequence, continental-scale estimates of the variability and trends in heavy precipitation based on daily precipitation might generally agree qualitatively but may exhibit significant quantitative differences. In Reference [5] a detailed review of this phenomenon is presented where it is noted that for the European continent, most results hint 
at a growing intensity of heavy precipitation over the last five decades. The changes in extreme precipitation volumes are also among the relevant problems of such areas as climate [6,7], shallow landslides [8], debris flows [9], and so forth. Therefore, tools for analyzing such observations are being improved, including precipitation reanalysis [10].

There are several schemes on how to detect the extreme precipitation volume and what can be understood as the extreme precipitation. It can be selected from the total precipitation as unique heavy rain or show, for instance, which contains the precipitation volume more then $95 \%$ or more volume among total precipitation for given period independently of whether rain was observed or not. Alternatively, the precipitation and its volume can be considered only during the wet period ignoring all dry spells. In this case the concept of extreme precipitation and its statistics will differ from its counterpart in the first case. The details are discussed for instance in Reference [11]. The classical extreme value approach based on Pickands-Balkema-de Haan theorem $[12,13]$ (the POT method) is used for solving such problems for precipitation data in References [14-16]. But sometimes it can lead to less accurate estimates of extreme values [17] than the methods proposed in the present paper (one possible alternative for estimating precipitation extremes based on the log-histospline was considered in Reference [18]). In the present paper, a rather reasonable approach to the unambiguous (algorithmic) determination of extreme or abnormally heavy daily and total precipitation within a wet period is proposed.

It is traditionally assumed that the duration of a wet period (the number of subsequent wet days) follows the geometric distribution (for example, see Reference [5]). But the sequence of dry and wet days is not only independent, it is also devoid of the Markov property [19]. Our approach introduces the negative binomial model for the duration of wet periods measured in days. This model demonstrates excellent fiting the numbers of successive wet days with the negative binomial distribution with shape parameter less than one, see References [20,21]. Numerous examples for dry and wet periods for different stations in Europe and Russia have been presented in Reference [22]. It provides a theoretical base for the determination of asymptotic approximations to the distributions of the maximum daily precipitation volume within a wet period and of the total precipitation volume for a wet period. The asymptotic distribution of the maximum daily precipitation volume within a wet period turns out to be a tempered Snedecor-Fisher distribution (i.e., the distribution of a positive power of a random variable with the Snedecor-Fisher distribution) whereas the total precipitation volume for a wet period turns out to be the gamma distribution. Both approximations appear to be very accurate. These asymptotic approximations are deduced using limit theorems for statistics constructed from samples with random sizes.

In this paper, two approaches are proposed to the definition of anomalously extremal precipitation. The first approach to the definition (and determination) of abnormally heavy daily precipitation is based on the tempered Snedecor-Fisher distribution (some methods of statistical estimation of its parameters will also be discussed). The second approach is based on the assumption that the total precipitation volume over a wet period has the gamma distribution. This assumption is theoretically justified by a version of the law of large numbers for sums of a random number of random variables in which the number of summands has the negative binomial distribution and is empirically substantiated by the statistical analysis of real data. Hence, the hypothesis that the total precipitation volume during a certain wet period is anomalously large can be formulated as the homogeneity hypothesis of a sample from the gamma distribution. Two equivalent tests are proposed for testing this hypothesis. One of them is based on the beta distribution whereas the second is based on the Snedecor-Fisher distribution. Both of these tests deal with the relative contribution of the total precipitation volume for a wet period to the considered set (sample) of successive wet periods. Within the second approach it is possible to introduce the notions of relatively abnormal and absolutely anomalous precipitation volumes. The results of the application of these tests to real data are presented yielding the conclusion that the intensity of wet periods with anomalously large precipitation volume increases. 
The proposed approaches have several important benefits First, estimates of total precipitation are weakly affected by the accuracy of the daily records and are less sensitive to missing values. Second, they are based on limit theorems of probability theorems that yield unambiguous asymptotic approximations which are used as adequate mathematical models. Third, these approaches provide unambiguous algorithms for the determination of extreme or anomalously heavy daily or total precipitation that do not involve statistical significance problems owing to the low occurrence of such (relatively rare) events.

Suggested methods improve the approach described in Reference [11], where an estimate of the fractional contribution from the wettest days to the total was developed which is less hampered by the limited number of wet days. This paper demonstrates that the proposed methods have a theoretical background and at the same time they are perfectly consistent with the real data. Our methods are also compared with previously suggested in Reference [11] on the same database. The comparison clearly showed that in many case the new approach gives more precise assessment for extremes and allows distinguishing the ordinary and extreme volume for precipitation more detailed. It is worth noting that all algorithms have been implemented as MATLAB software tools.

The paper is organized as follows. In Section 2 mathematical models to derive statistical tests for the extreme precipitation events are introduced. The tempered Snedecor-Fisher distribution as an asymptotic approximation to the maximum daily precipitation volume within a wet period and some corresponding analytic properties are presented. Two equivalent statistical tests for a total precipitation volume over a wet period to be abnormally large based on testing the homogeneity hypothesis of a sample from the gamma distribution are introduced. Section 3 describes the application of the proposed models and algorithms to real data using precipitation observations in Potsdam and Elista in about 60 years. Section 4 is devoted to the main conclusions of the work.

\section{Mathematical Models to Derive Statistical Tests for Precipitation Volume to Be Anomalous Large}

At the beginning of this section some notations that will be used below are introduced. All the random variables under consideration are defined on the same probability space $(\Omega, \mathfrak{F}, \mathbb{P})$. The results are expounded in terms of random variables with the corresponding distributions. The symbol $\stackrel{d}{=}$ denotes the coincidence of distributions.

Let $G_{r, \lambda}$ be a random variable having the gamma distribution with shape parameter $r>0$ and scale parameter $\lambda>0$ :

$$
\mathbb{P}\left(G_{r, \lambda}<x\right)=\int_{0}^{x} \frac{\lambda^{r}}{\Gamma(r)} z^{r-1} e^{-\lambda z} d z, \quad x \geqslant 0,
$$

Let $W_{\gamma}$ be a random variable with the Weibull distribution with the distribution function $\left[1-e^{-x^{\gamma}}\right] \mathbf{1}(x \geqslant 0)(\mathbf{1}(A)$ is the indicator function of a set $A)$. The distribution of the random variable $|X|$, where $X$ is a random variable with the standard normal distribution function, is a folded normal $(x \geqslant 0)$ :

$$
\mathbf{P}(|X|<x)=2 \Phi(x)-1
$$

Let $S_{\alpha, 1}$ and $S_{\alpha, 1}^{\prime}(0<\alpha<1)$ be independent and identically-distributed random variables with the same strictly stable distribution [23]. So, the density $v_{\alpha}(x)$ of the random variable $R_{\alpha}=S_{\alpha, 1} / S_{\alpha, 1}^{\prime}$ can be represented [24] as follows $(x>0)$ :

$$
v_{\alpha}(x)=\frac{\sin (\pi \alpha) x^{\alpha-1}}{\pi\left[1+x^{2 \alpha}+2 x^{\alpha} \cos (\pi \alpha)\right]} .
$$


2.1. The Tempered Snedecor-Fisher Distribution as an Asymptotic Approximation to the Maximum Daily Precipitation Volume Within a Wet Period

As it has been demonstrated in Reference [25], the asymptotic probability distribution of extremal daily precipitation within a wet period can be represented as follows (here $r>0, \lambda>0$, and $\gamma>0$ ):

$$
F(x ; r, \lambda, \gamma)=\left(\frac{\lambda x^{\gamma}}{1+\lambda x^{\gamma}}\right)^{r}, x \geqslant 0
$$

Moreover, the theoretical conditions of limit theorems correspond with the real data (in sense of fitting Pareto distribution, see Reference [26]). The function (3) is a scale mixture of the Fréchet (inverse Weibull) distribution. It can be demonstrated for a random variable $M_{r, \gamma, \lambda}$ with a distribution function $F(x ; r, \lambda, \gamma)$ that

$$
M_{r, \gamma, \lambda} \stackrel{d}{=}\left(\frac{Q_{r, 1}}{\lambda r}\right)^{1 / \gamma}
$$

that is, the distribution of the random variable $M_{r, \gamma, \lambda}$ up to a non-random scale factor coincides with that of the positive power of a random variable with the Snedecor-Fisher distribution. In other words, the distribution function $F(x ; r, \lambda, \gamma)$ (3) up to a power transformation of the argument $x$ coincides with the Snedecor-Fisher distribution function. In statistics, distributions with arguments subjected to the power transformation are conventionally called tempered. Therefore, the distribution $F(x ; r, \lambda, \gamma)$ can be called tempered Snedecor-Fisher distribution. Some properties of the distribution of the random value $M_{r, \gamma, \lambda}$ were discussed in [26]. In particular, it was shown that the limit distribution (3) can be represented as a scale mixture of exponential or stable or Weibull or Pareto or folded normal laws $(r \in(0,1], \gamma \in(0,1], \lambda>0)$ :

$$
M_{r, \gamma, \lambda} \stackrel{d}{=} \frac{G_{r, \lambda}^{1 / \gamma} S_{\gamma, 1}}{W_{1}} \stackrel{d}{=} \frac{W_{\gamma}}{W_{\gamma}^{\prime}} \cdot \frac{1}{Z_{r, \lambda}^{1 / \gamma}} \stackrel{d}{=} W_{1} \cdot \frac{R_{\gamma}}{W_{1}^{\prime} Z_{r, \lambda}^{1 / \gamma}} \stackrel{d}{=} \frac{\Pi R_{\gamma}}{Z_{r, \lambda}^{1 / \gamma}} \stackrel{d X \mid \sqrt{2 W_{1}} R_{\gamma}}{=} \frac{\mid X Z_{r, \lambda}^{\prime / \gamma}}{Z^{1 / \gamma}}
$$

where $W_{\gamma} \stackrel{d}{=} W_{\gamma}^{\prime}, W_{1} \stackrel{d}{=} W_{1}^{\prime}$, the random variable $R_{\gamma}$ has the density (2), the random variable $\Pi$ has the Pareto distribution $\left(\mathbb{P}(\Pi>x)=(x+1)^{-1}, x \geqslant 0\right)$, and in each term the involved random variables are independent.

It should be mentioned that the same mathematical reasoning can be used for the determination of the asymptotic distribution of the maximum daily precipitation within $m$ wet periods with arbitrary finite $m \in \mathbb{N}$. Indeed, fix arbitrary positive $r_{1}, \ldots, r_{m}$ and $p \in(0,1)$. Let $N_{r_{1}, p}^{(1)}, \ldots, N_{r_{m}, p}^{(m)}$ be independent random variables having the negative binomial distributions with parameters $r_{j}, p, j=1, \ldots, m$, respectively. By the consideration of characteristic functions it can be easily verified that

$$
N_{r_{1}, p}^{(1)}+\ldots+N_{r_{m}, p}^{(m)} \stackrel{d}{=} N_{r, p}
$$

where $r=r_{1}+\ldots+r_{m}$. If all $r_{j}$ coincide, then $r=m r_{1}$ and in accordance with relation (4), the asymptotic distribution of the maximum daily precipitation within $m$ wet periods has the form $(x \geqslant 0)$

$$
F^{(m)}(x ; r, \lambda, \gamma)=F\left(x ; m r_{1}, \lambda, \gamma\right)=\left(\frac{\lambda x^{\gamma}}{1+\lambda x^{\gamma}}\right)^{m r_{1}}
$$

And if now $m$ infinitely increases and simultaneously $\lambda$ changes as $\lambda=c m, c \in(0, \infty)$, then, obviously,

$$
\lim _{m \rightarrow \infty} F^{(m)}(x ; r, \lambda, \gamma)=\lim _{m \rightarrow \infty} F\left(x ; m r_{1}, c m, \gamma\right)=e^{-\mu x^{-\gamma}}
$$

with $\mu=\left(c r_{1}\right)^{-1}$, that is, the distribution function

$F^{(m)}(x ; r, \lambda, \gamma)$ of the maximum daily precipitation within $m$ wet periods turns into the classical Fréchet distribution. 
This model makes it possible to propose the following approach to the definition (and determination) of an anomalously heavy daily precipitation volume. The grounds for this approach is an obvious observation that if $X_{1}, X_{2}, \ldots, X_{N}$ is a sample of $N$ positive observations, then with finite (possibly, random) $N$, among $X_{i}{ }^{\prime}$ s there is always an extreme observation, say, $X_{1}$, such that $X_{1} \geqslant X_{i}, i=1,2, \ldots, N$. Two cases are possible: $X_{1}$ is a 'typical' observation and its extreme character is conditioned by purely stochastic circumstances (there must be an extreme observation within a finite homogeneous sample) and $X_{1}$ is abnormally large so that it is an outlier and its extreme character is due to some exogenous factors. It follows from (3) that the distribution of $X_{1}$ in the first case is the tempered Snedecor-Fisher distribution. Therefore, if $X_{1}$ exceeds a certain (pre-defined) quantile of this distribution, then it is regarded as suspicious to be an outlier. The examples will be demonstrated in Section 3.2.

\subsection{The Algorithms of Statistical Fitting of the Tempered Snedecor-Fisher Distribution Model}

In this section the algorithms and corresponding formulas for a statistical estimation of the parameters $r, \lambda$ and $\gamma$ of the tempered Snedecor-Fisher distribution (3) are briefly given (for details, see Reference [26]).

Let $\left\{X_{i, j}\right\}, i=1, \ldots, m, j=1, \ldots, m_{i}$, be the precipitation volumes on the $j$ th day of the $i$ th wet sequence. Let $X_{(1)}^{*}, \ldots, X_{(m)}^{*}$ be order statistics constructed from the sample $X_{1}^{*}, \ldots, X_{m}^{*}$, where $X_{k}^{*}=\max \left\{X_{k, 1}, \ldots, X_{k, m_{k}}\right\}$. The unknown parameters $r, \lambda$ and $\gamma$ can be found as a solution of a following system of equations (for fixed values $p_{1}, p_{2}$ and $p_{3}, 0<p_{1}<p_{2}<p_{3}<1$ ):

$$
X_{\left(\left[m p_{k}\right]\right)}^{*}=\left(\frac{p_{k}^{1 / r}}{\lambda-\lambda p_{k}^{1 / r}}\right)^{1 / \gamma}, \quad k=1,2,3
$$

(here the symbol $[a]$ denotes the integer part of a number $a$ ).

Proposition 1. The values of parameters $\gamma$ and $\lambda$ can be estimated as follows:

$$
\begin{gathered}
\widetilde{\gamma}_{q}=\frac{\frac{1}{r}\left(\log p_{1}-\log p_{3}\right)+\log \left(1-p_{3}^{\frac{1}{r}}\right)-\log \left(1-p_{1}^{\frac{1}{r}}\right)}{\log X_{\left(\left[m p_{1}\right]\right)}^{*}-\log X_{\left(\left[m p_{3}\right]\right)}^{*}}, \\
\widetilde{\lambda}_{q}=\frac{p_{2}^{\frac{1}{r}}}{\left(1-p_{2}^{\frac{1}{r}}\right)\left(X_{\left(\left[m p_{2}\right]\right)}^{*}\right)^{\gamma}} .
\end{gathered}
$$

The expressions (6) and (7) are obtained as solutions to the system of Equation (5) while the parameter $r$ is determined numerically.

Proposition 2. If the value of parameter $r$ is estimated as a corresponding parameter of the negative binomial distribution, least squares estimates of parameters $\gamma$ and $\lambda$ are as follows:

$$
\begin{aligned}
\widehat{\gamma}_{L S}= & \sum_{j=1}^{m-1} \log X_{(j)}^{*}\left(\left(\log \frac{j^{1 / r}}{m^{1 / r}-j^{1 / r}}\right)^{m-1}-\sum_{k=1}^{m-1} \log \frac{k^{1 / r}}{m^{1 / r}-k^{1 / r}}\right) \times \\
& \times\left((m-1) \sum_{j=1}^{m-1}\left(\log X_{(j)}^{*}\right)^{2}-\left(\sum_{j=1}^{m-1} \log X_{(j)}^{*}\right)^{2}\right)^{-1}, \\
\widehat{\lambda}_{L S}= & \exp \left\{\frac{1}{m-1}\left(\sum_{j=1}^{m-1} \log \frac{j^{1 / r}}{m^{1 / r}-j^{1 / r}}-\widehat{\gamma}_{L S} \sum_{j=1}^{m-1} \log X_{(j)}^{*}\right)\right\} .
\end{aligned}
$$


The derivation of the Formulas (8) and (9) is based on a minimization of the discrepancy between the empirical and model distribution functions using the least squares techniques.

The examples of fitting tempered Snedecor-Fisher distribution to the real data will be discussed in Section 3.1. The examples of determining an extreme daily precipitation volume based on quantiles of this distribution will be demonstrated in Section 3.2 using precipitation observations in Potsdam and Elista from 1950 to 2007.

2.3. The Tests for a Total Precipitation Volume to Be Anomalously Extremal Based on the Homogeneity Test of a Sample From the Gamma Distribution

Here some algorithms of testing the hypotheses that a total precipitation volume during a wet period is anomalously extremal within a certain time horizon are proposed. Moreover, this approach makes it possible to consider relatively anomalously extremal volumes and absolutely anomalously extremal volumes for a given time horizon.

Let $m \in \mathbb{N}$ and $G_{r, \mu}^{(1)}, G_{r, \mu}^{(2)}, \ldots, G_{r, \mu}^{(m)}$-be independent random variables having the same gamma distribution with shape parameter $r>0$ and scale parameter $\mu>0$. In Reference [11] it was suggested to use the distribution of the ratio

$$
R^{*}=\frac{G_{r, \mu}^{(1)}}{G_{r, \mu}^{(1)}+G_{r, \mu}^{(2)}+\ldots+G_{r, \mu}^{(m)}} \stackrel{d}{=} \frac{G_{r, 1}^{(1)}}{G_{r, 1}^{(1)}+G_{r, 1}^{(2)}+\ldots+G_{r, 1}^{(m)}}
$$

as a heuristic model of the distribution of the extremely large precipitation volume based on the assumption that fluctuations of daily precipitation follow the gamma distribution. The gamma model for the distribution of daily precipitation volume is less adequate than the Pareto one [26]. Here we will modify the technique proposed in Reference [11] and make it more adequate and justified.

Let $X_{1}, X_{2}, \ldots$ be daily precipitation volumes on wet days. For $k \in \mathbb{N}$ denote $S_{k}=X_{1}+\ldots+X_{k}$. The statistical analysis of the observed data shows that the average daily precipitation volume on wet days is finite:

$$
\frac{1}{n} \sum_{j=1}^{n} X_{j} \Longrightarrow a \in(0, \infty)
$$

Here the symbol $\Longrightarrow$ denotes the convergence in distribution.

Figure 1 illustrates the stabilization of the cumulative averages of daily precipitation volumes as $n$ grows in Potsdam (continuous line) and Elista (dash line), and thus, the practical validity of assumption (10). It should be emphasized that $X_{1}, X_{2}, \ldots$ can be dependent.

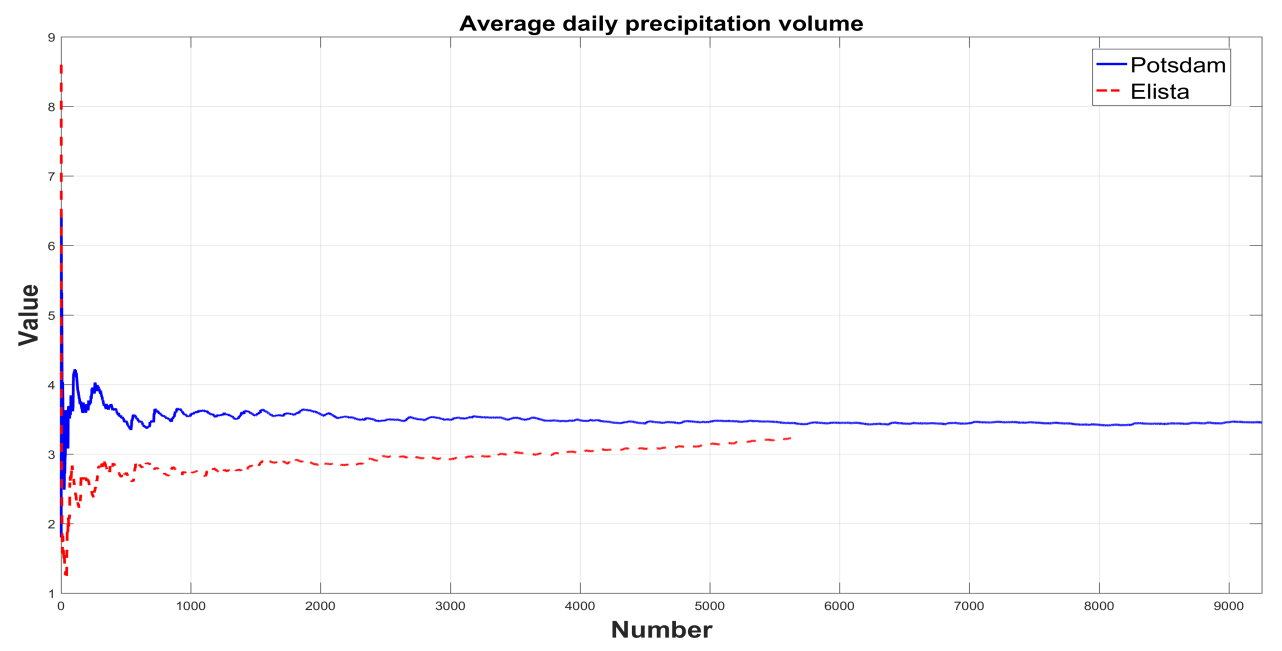

Figure 1. Stabilization of the cumulative averages of daily precipitation volumes as $n$ grows in Potsdam (continuous line) and Elista (dash line). 
Let $r>0, \mu>0, q \in(0,1), n \in \mathbb{N}$. Let the random variable $N_{r, p_{n}}$ have the negative binomial distribution with parameters $r$ and $p_{n}=\min \{q, \mu / n\}$. Using the properties of characteristic functions it is easy to make sure that

$$
n^{-1} N_{r, p_{n}} \Longrightarrow G_{r, \mu} \stackrel{d}{=} \frac{1}{\mu} G_{r, 1}
$$

as $n \rightarrow \infty$. So, the following analog of the law of large numbers for negative binomial random sums can be obtained. It can be actually regarded as a generalization of the Rényi theorem concerning the rarefaction of renewal processes.

Theorem 1. Assume that the daily precipitation volumes on wet days $X_{1}, X_{2}, \ldots$ satisfy condition (10). Let the numbers $r>0, q \in(0,1)$ and $\mu>0$ be arbitrary. For each $n \in \mathbb{N}$, let the random variable $N_{r, p_{n}}$ have the negative binomial distribution with parameters $r$ and $p_{n}=\min \{q, \mu / n\}$. Assume that the random variables $N_{r, p_{n}}$ are independent of the sequence $X_{1}, X_{2}, \ldots$ Then

$$
n^{-1} \sum_{j=1}^{N_{r, p_{n}}} X_{j} \Longrightarrow a G_{r, \mu} \stackrel{d}{=} \frac{a}{\mu} G_{r, 1} \quad \text { as } n \rightarrow \infty .
$$

Proof. Consider a sequence of random variables $\left\{W_{n}=\sum_{j=1}^{n} X_{j}\right\}, n \in \mathbb{N}$. Let us introduce the following notations:

$$
b_{n}=d_{n}=n, \quad N_{n}=N_{r, p_{n}}, \quad W=a, \quad N=\frac{1}{\mu} G_{r, 1},
$$

where for every $n \in \mathbb{N}$ the random variable $N_{n}$ is independent of the sequence $\left\{W_{n}\right\}$. Then, the expressions

$$
b_{n}^{-1} W_{n} \Longrightarrow W, \quad d_{n}^{-1} b_{N_{n}} \Longrightarrow N
$$

take place as $n \rightarrow \infty$ (see (10) and (11), respectively). As all the conditions of the theorem for random sequences with independent random indices $[27,28]$ are satisfied, we obtain the following expression:

$$
d_{n}^{-1} W_{N_{n}} \Longrightarrow W \cdot N \quad \text { as } n \rightarrow \infty
$$

Taking into account above-mentioned notations, one can conclude that relation (12) holds.

Therefore, with the account of the excellent fit of the negative binomial model for the duration of a wet period [26] with rather small $p_{n}$, the gamma distribution can be regarded as an adequate and theoretically well-based model for the total precipitation volume during a (long enough) wet period. This theoretical conclusion based on the negative binomial model for the distribution of duration of a wet period is vividly illustrated by the empirical data as shown on Figure 2 where the histograms of total precipitation volumes in Potsdam and Elista and the fitted gamma distributions are shown. For comparison, the densities of the best generalized Pareto distributions are also presented. It can be seen that even the best fitted Pareto distributions demonstrate worse fit than the gamma distribution.

Let $m \in \mathbb{N}$ and $G_{r, \mu}^{(1)}, G_{r, \mu}^{(2)}, \ldots, G_{r, \mu}^{(m)}$ be independent random variables having the same gamma distribution with parameters $r>0$ and $\mu>0$. Consider the relative contribution of the random variable $G_{r, \mu}^{(1)}$ to the sum $G_{r, \mu}^{(1)}+G_{r, \mu}^{(2)}+\ldots+G_{r, \mu}^{(m)}$ :

$$
\begin{aligned}
& R=\frac{G_{r, \mu}^{(1)}}{G_{r, \mu}^{(1)}+G_{r, \mu}^{(2)}+\ldots+G_{r, \mu}^{(m)}} \stackrel{d}{=} \frac{G_{r, 1}^{(1)}}{G_{r, 1}^{(1)}+G_{r, 1}^{(2)}+\ldots+G_{r, 1}^{(m)}} \stackrel{d}{=} \\
& \stackrel{d}{=}\left(1+\frac{1}{G_{r, 1}^{(1)}}\left(G_{r, 1}^{(2)}+\ldots+G_{r, 1}^{(m)}\right)\right)^{-1} \stackrel{d}{=}\left(1+\frac{G_{(m-1) r, 1}}{G_{r, 1}}\right)^{-1},
\end{aligned}
$$


where the gamma-distributed random variables on the right hand side are independent. So, the random variable $R$ characterizes the relative precipitation volume for one (long enough) wet period with respect to the total precipitation volume registered for $m$ wet periods.

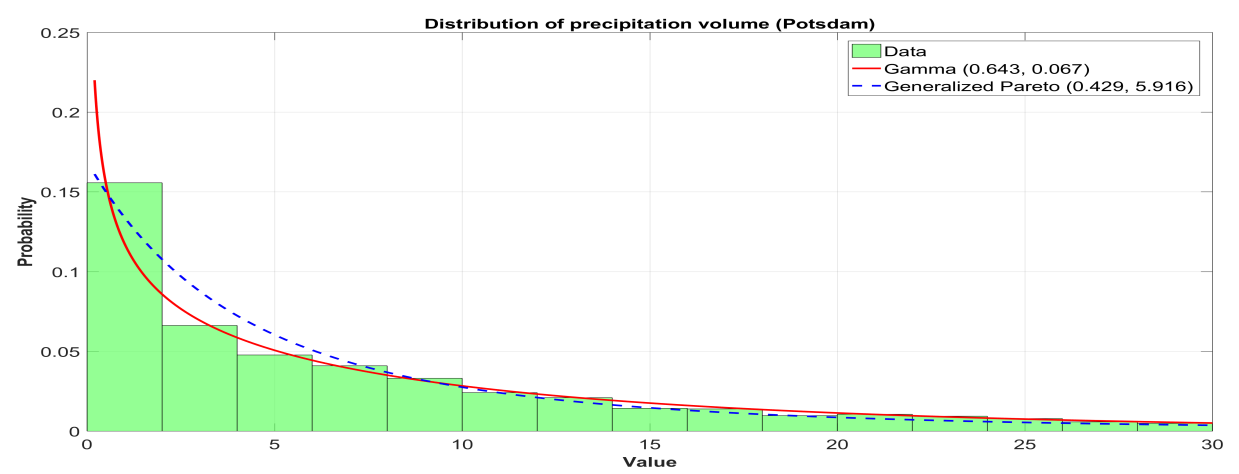

(a)

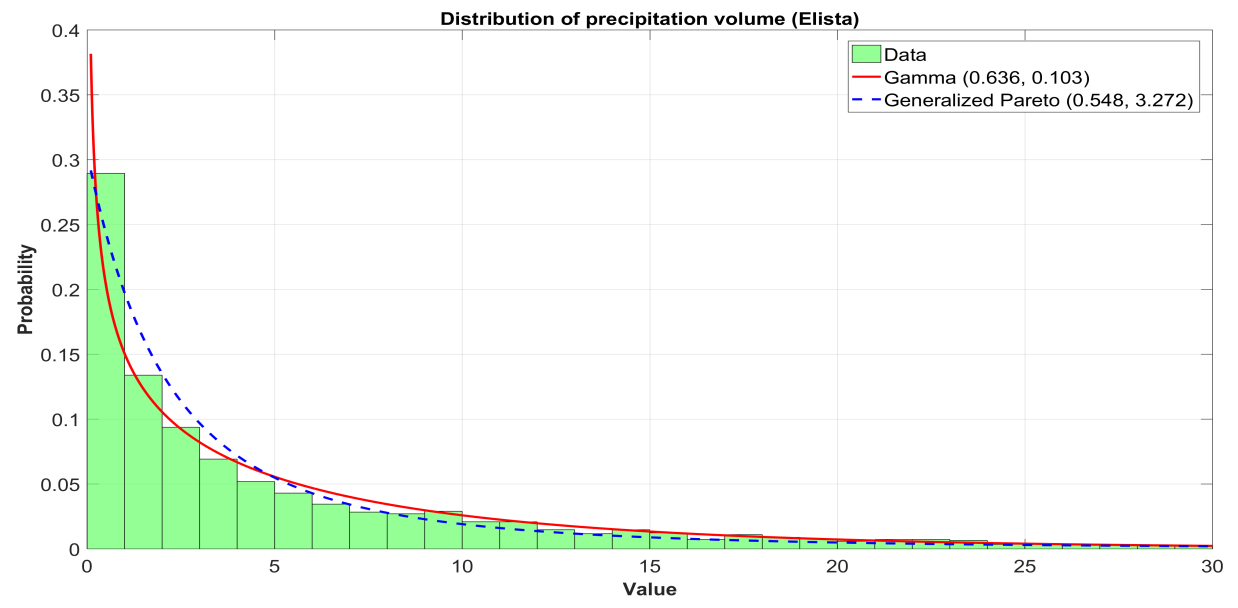

(b)

Figure 2. The histograms of total precipitation volumes in Potsdam (a) and Elista (b) and the fitted gamma and generalized Pareto distributions.

The distribution of the random variable $R$ is completely determined by the distribution of the ratio of two independent gamma-distributed random variables. To find the latter, denote $k=(m-1) r$ and obtain

$$
\frac{G_{k, 1}}{G_{r, 1}}=\frac{k}{r} \cdot\left(\frac{r}{k} \cdot \frac{G_{k, 1}}{G_{r, 1}}\right) \stackrel{d}{=} \frac{k}{r} \cdot Q_{k, r}
$$

where $Q_{k, r}$ is the random variable having the Snedecor-Fisher distribution determined for $k>0, r>0$ by the Lebesgue density

$$
f_{k, r}(x)=\frac{\Gamma(k+r)}{\Gamma(k) \Gamma(r)}\left(\frac{k}{r}\right)^{k} \frac{x^{k-1}}{\left(1+\frac{k}{r} x\right)^{k+r}}, \quad x \geqslant 0,
$$

(as is known, $Q_{k, r} \stackrel{d}{=} r G_{k, 1}\left(k G_{r, 1}\right)^{-1}$, where the random variables $G_{k, 1}$ and $G_{r, 1}$ are independent (see, e.g., Reference [29], p. 32)). It is worth noting that the particular value of the scale parameter is insignificant. For convenience, it is assumed equal to one.

So, $R \stackrel{d}{=}\left(1+\frac{k}{r} Q_{k, r}\right)^{-1}$, and, as is easily made sure by standard calculation using (14), the distribution of the random variable $R$ is determined by the density

$$
p(x ; k, r)=\frac{\Gamma(k+r)}{\Gamma(r) \Gamma(k)}(1-x)^{k-1} x^{r-1}, \quad 0 \leqslant x \leqslant 1,
$$


that is, it is the beta distribution with parameters $k=(m-1) r$ and $r$.

Then the test for the homogeneity of an independent sample of size $m$ consisting of the gamma-distributed observations of total precipitation volumes during $m$ wet periods with known $\gamma$ based on the random variable $R$ looks as follows. Let $V_{1}, \ldots, V_{m}$ be the total precipitation volumes during $m$ wet periods and, moreover, $V_{1} \geqslant V_{j}$ for all $j \geqslant 2$. Calculate the quantity

$$
S R=\frac{V_{1}}{V_{1}+\ldots+V_{m}}
$$

( $S R$ means "Sample $R$ "). From what was said above it follows that under the hypothesis $H_{0}$ : "the precipitation volume $V_{1}$ under consideration is not anomalously large" the random variable $S R$ has the beta distribution with parameters $k=(m-1) r$ and $r$. Let $\alpha \in(0,1)$ be a small number, $\beta_{k, r}(1-\alpha)$ be the $(1-\alpha)$-quantile of the beta distribution with parameters $k=(m-1) r$ and $r$. If $S R>\beta_{k, r}(1-\alpha)$, then the hypothesis $H_{0}$ must be rejected, that is, the volume $V_{1}$ of precipitation during one wet period must be regarded as anomalously large. Moreover, the probability of erroneous rejection of $H_{0}$ is equal to $\alpha$.

Instead of $R$ (13), the quantity

$$
R_{0}=\frac{(m-1) G_{r, \mu}^{(1)}}{G_{r, \mu}^{(2)}+\ldots+G_{r, \mu}^{(m)}} \stackrel{d}{=} \frac{k}{r} \frac{G_{r, \mu}}{G_{k, \mu}} \stackrel{d}{=} \frac{k}{r} \frac{G_{r, 1}}{G_{k, 1}} \stackrel{d}{=} Q_{r, k}
$$

can be considered. Then, as is easily seen, the random variables $R$ and $R_{0}$ are related by the one-to-one correspondence

$$
R=\frac{R_{0}}{m-1+R_{0}} \text { or } R_{0}=\frac{(m-1) R}{1-R},
$$

so that the homogeneity test for a sample from the gamma distribution equivalent to the one described above and, correspondingly, the test for a precipitation volume during a wet period to be anomalously large, can be based on the random variable $R_{0}$ which has the Snedecor-Fisher distribution with parameters $r$ and $k=(m-1) r$.

Namely, again let $V_{1}, \ldots, V_{m}$ be the total precipitation volumes during $m$ wet periods and, moreover, $V_{1} \geqslant V_{j}$ for all $j \geqslant 2$. Calculate the quantity

$$
S R_{0}=\frac{(m-1) V_{1}}{V_{2}+\ldots+V_{m}}
$$

( $S R_{0}$ means "Sample $\left.R_{0}{ }^{\prime \prime}\right)$. From what was said above it follows that under the hypothesis $H_{0}$ : "the precipitation volume $V_{1}$ under consideration is not anomalously large" the random variable $S R$ has the Snedecor-Fisher distribution with parameters $r$ and $k=(m-1) r$. Let $\alpha \in(0,1)$ be a small number, $q_{r, k}(1-\alpha)$ be the $(1-\alpha)$-quantile of the Snedecor-Fisher distribution with parameters $r$ and $k=(m-1) r$. If $S R_{0}>q_{r, k}(1-\alpha)$, then the hypothesis $H_{0}$ must be rejected, that is, the volume $V_{1}$ of precipitation during one wet period must be regarded as anomalously large. Moreover, the probability of erroneous rejection of $H_{0}$ is equal to $\alpha$.

Let $l$ be a natural number, $1 \leqslant l<m$. It is worth noting that, unlike the test based on the statistic $R$, the test based on $R_{0}$ can be modified for testing the hypothesis $H_{0}^{\prime}$ : "the precipitation volumes $V_{i_{1}}, V_{i_{2}}, \ldots, V_{i_{l}}$ do not make an anomalously large cumulative contribution to the total precipitation volume $V_{1}+\ldots+V_{m}{ }^{\prime \prime}$. For this purpose denote

$$
T_{l}=V_{i_{1}}+V_{i_{2}}+\ldots+V_{i_{l}}, \quad T=V_{1}+V_{2}+\ldots+V_{m}
$$

and consider the quantity

$$
S R_{0}^{\prime}=\frac{(m-l) T_{l}}{l\left(T-T_{l}\right)}
$$


In the same way as it was done above, it is easy to make sure that

$$
S R_{0}^{\prime} \stackrel{d}{=} \frac{(m-l) G_{l r, l}}{l G_{(m-l) r, 1}} \stackrel{d}{=} Q_{l r,(m-l) r}
$$

Let $\alpha \in(0,1)$ be a small number, $q_{l r,(m-1) r}(1-\alpha)$ be the $(1-\alpha)$-quantile of the Snedecor-Fisher distribution with parameters $l r$ and $k=(m-l) r$. If $S R_{0}^{\prime}>q_{l r,(m-l) r}(1-\alpha)$, then the hypothesis $H_{0}^{\prime}$ must be rejected, that is, the cumulative contribution of the precipitation volumes $V_{i_{1}}, V_{i_{2}}, \ldots, V_{i_{l}}$ into the total precipitation volume $V_{1}+\ldots+V_{m}$ must be regarded as anomalously large. Moreover, the probability of erroneous rejection of $H_{0}^{\prime}$ is equal to $\alpha$.

The examples of application of the test for a total precipitation volume within a wet period to be anomalously large will be discussed in Section 3.4 using precipitation observations in Potsdam and Elista from 1950 to 2007.

\section{The Results of the Analysis of Real Data}

\subsection{Statistical Fitting of the Tempered Snedecor-Fisher Distribution Model to Real Data}

The numerical results of estimation of the parameters of daily precipitation in Potsdam and Elista from 1950 to 2009 using both algorithms described in Propositions 1 and 2 (see Section 2.2) are presented in Tables 1 and 2. The first column indicates the censoring threshold: since the tempered Snedecor-Fisher distribution is an asymptotic model which is assumed to be more adequate with small "success probability," the estimates were constructed from differently censored samples which contain only those wet periods whose duration is no less than the specified threshold.

Table 1. Estimation of the parameters of daily precipitation in Potsdam ( $r=0.847)$.

\begin{tabular}{cccccccc}
\hline Minimum Duration & Sample Size & $\mathbf{D}_{\mathbf{q}}$ & $\mathbf{D}_{\mathbf{L S}}$ & $\widetilde{\lambda}_{\mathbf{q}}$ & $\widehat{\lambda}_{\mathbf{q}}$ & $\widetilde{\gamma}_{\mathbf{L S}}$ & $\widehat{\gamma}_{\mathbf{L S}}$ \\
\hline 1 & 3323 & 0.09 & 0.092 & 0.169 & 0.212 & 1.18 & 1.29 \\
2 & 2066 & 0.045 & 0.065 & 0.0383 & 0.054 & 1.755 & 1.71 \\
3 & 1282 & 0.031 & 0.041 & 0.01 & 0.013 & 2.261 & 2.183 \\
4 & 862 & 0.026 & 0.027 & 0.0049 & 0.0045 & 2.449 & 2.524 \\
6 & 384 & 0.025 & 0.026 & 0.0015 & 0.0012 & 2.822 & 2.949 \\
8 & 163 & 0.04 & 0.045 & 0.0007 & 0.0005 & 3.174 & 3.255 \\
10 & 73 & 0.041 & 0.042 & 0.0003 & 0.0003 & 3.385 & 3.352 \\
15 & 12 & 0.13 & 0.09 & 0.0014 & 0.0009 & 2.667 & 2.973 \\
\hline
\end{tabular}

Table 2. Estimation of the parameters of daily precipitation in Elista $(r=0.876)$.

\begin{tabular}{cccccccc}
\hline Minimum Duration & Sample Size & $\mathbf{D}_{\mathbf{q}}$ & $\mathbf{D}_{\mathbf{L S}}$ & $\widetilde{\lambda}_{\mathbf{q}}$ & $\widehat{\lambda}_{\mathbf{q}}$ & $\widetilde{\gamma}_{\mathbf{L S}}$ & $\widehat{\gamma}_{\text {LS }}$ \\
\hline 1 & 2937 & 0.06 & 0.06 & 0.361 & 0.349 & 1.053 & 1.263 \\
2 & 1374 & 0.049 & 0.055 & 0.108 & 0.101 & 1.424 & 1.574 \\
3 & 656 & 0.041 & 0.045 & 0.0454 & 0.0376 & 1.707 & 1.9 \\
4 & 319 & 0.051 & 0.06 & 0.0234 & 0.0273 & 1.891 & 1.94 \\
6 & 77 & 0.07 & 0.075 & 0.0181 & 0.0144 & 2.011 & 2.186 \\
7 & 42 & 0.15 & 0.01 & 0.0197 & 0.0207 & 1.983 & 2.179 \\
8 & 22 & 0.12 & 0.14 & 0.014 & 0.0358 & 2.01 & 1.764 \\
10 & 10 & 0.17 & 0.16 & 0.0136 & 0.0375 & 2.163 & 1.802 \\
\hline
\end{tabular}

The second column contains the correspondingly censored sample size. The third and fourth columns contain the sup-norm discrepancies $D_{q}$ and $D_{L S}$ between the empirical $\widehat{F}(x)$ and 
fitted tempered Snedecor-Fisher distributions $F_{S F}^{(q)}(x)$ and $F_{S F}^{(L S)}(x)$ for two types of estimators (quantile (6), (7) and least squares (8), (9)) described above. The quantities $D_{q}$ and $D_{L S}$ are as follows:

$$
D_{q}=\max _{x \in \mathbf{X}}\left|\widehat{F}(x)-F_{S F}^{(q)}(x)\right|, \quad D_{L S}=\max _{x \in \mathbf{X}}\left|\widehat{F}(x)-F_{S F}^{(L S)}(x)\right|,
$$

where $\mathbf{X}=\left(X_{1}, \ldots, X_{n}\right)$ is a sample. The rest columns contain the corresponding values of the parameters estimated by these two methods.

According to Tables 1 and 2, the best accuracy is attained when the censoring threshold equals 3 days for Elista and 5-6 days for Potsdam. The least squares method leads to the more accurate estimates. Figures 3 and 4 demonstrate examples of fitted tempered Snedecor-Fisher distribution to precipitation in Potsdam and Elista, respectively.
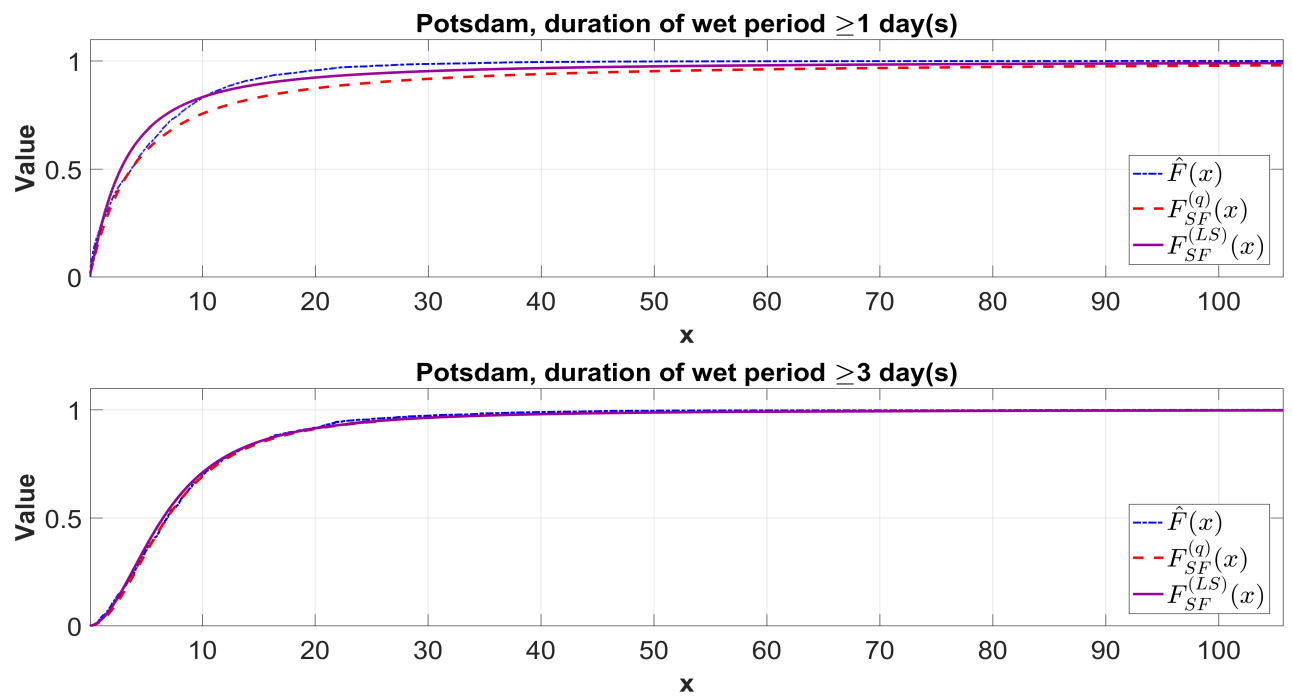

Figure 3. Fitted tempered Snedecor-Fisher distribution to precipitation in Potsdam.

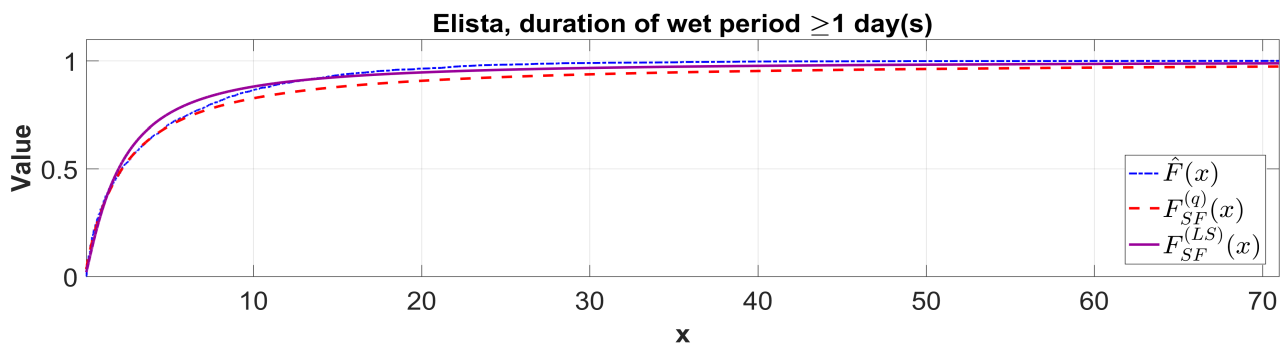

Elista, duration of wet period $\geq 3$ day(s)

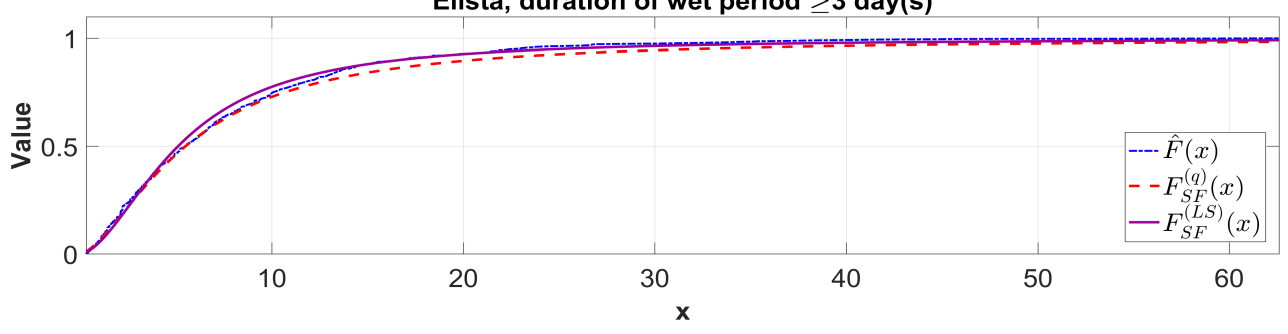

Figure 4. Fitted tempered Snedecor-Fisher distribution to precipitation in Elista.

3.2. Determining an Extreme Daily Precipitation Volume Based on Quantiles of the Tempered Snedecor-Fisher Distribution

In applied problems dealing with extreme values there is a common tradition which, possibly, has already become a prejudice, that statistical regularities in the behavior of extreme values necessarily 
obey one of well-known three types of extreme value distributions. In general, this is certainly so, if the sample size is very large, that is, the time horizon under consideration is very wide. In other words, the models based on the extreme value distributions have asymptotic character. However, in real practice, when the sample size is finite and the extreme values of the process under consideration are studied on the time horizon of a moderate length, the classical extreme value distributions may turn out to be inadequate models. In these situations a more thorough analysis may generate other models which appear to be considerably more adequate. This is exactly the case discussed in the present paper. Methodically, this approach is similar to the classical techniques of dealing with extreme observations [30]. The novelty of the proposed method is in a more accurate specification of the distribution of extreme daily precipitation which turned out to be the tempered Snedecor-Fisher distribution. Figure 5 demonstrates the algorithm of determination of an anomalously heavy daily precipitation.

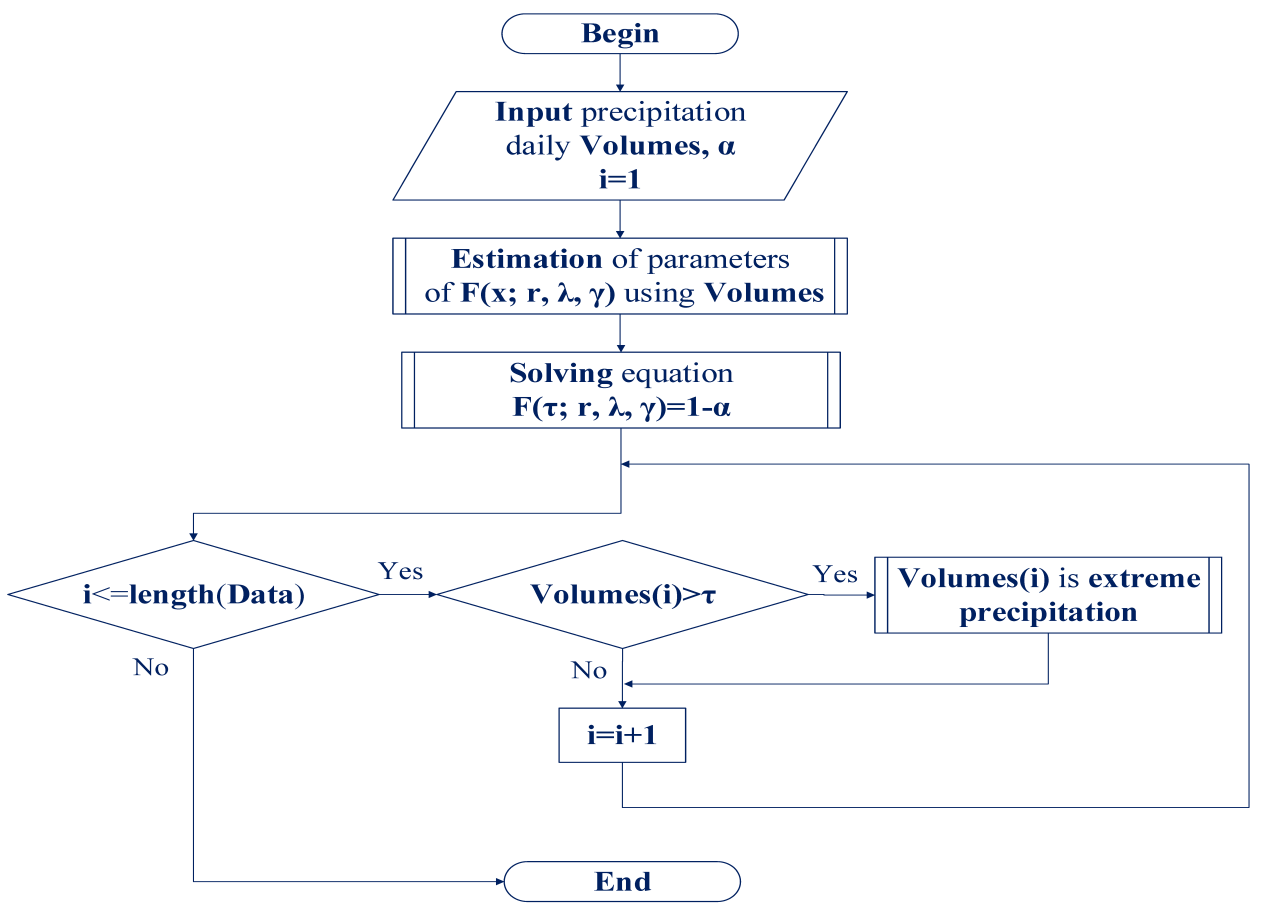

Figure 5. The algorithm of determination of an anomalously heavy daily precipitation.

It is easy to see that the the probability of the error of the first kind (occurring in the case where a regularly large maximum value is erroneously recognized as an anomalously large outlier) for this test is approximately equal to $\alpha$, it is a small fixed positive number.

The application of this test to real data is illustrated by Figures 6 and 7. On these figures the lower horizontal line corresponds to the threshold equal to the quantile of the fitted tempered Snedecor-Fisher distribution of order 0.9. The middle and upper lines correspond to the quantiles of orders 0.95 and 0.99 , respectively. Figure 6 contains all data. For the sake of vividness, on Figure 7 only one, maximum, daily precipitation is exposed for each wet period.

From Figure 7 it is seen that during 58 years (from 1950 to 2007) in Potsdam there were 13 wet periods containing anomalously heavy maximum daily precipitation volumes (at $99 \%$ threshold) and 69 wet periods containing anomalously heavy maximum daily precipitation volumes (at 95\% threshold). Other maxima were 'regular.' 


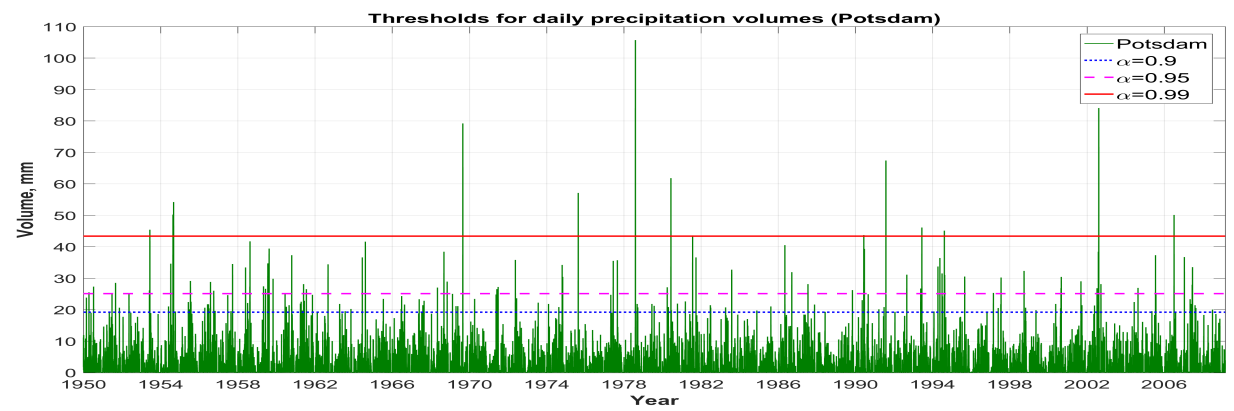

(a)

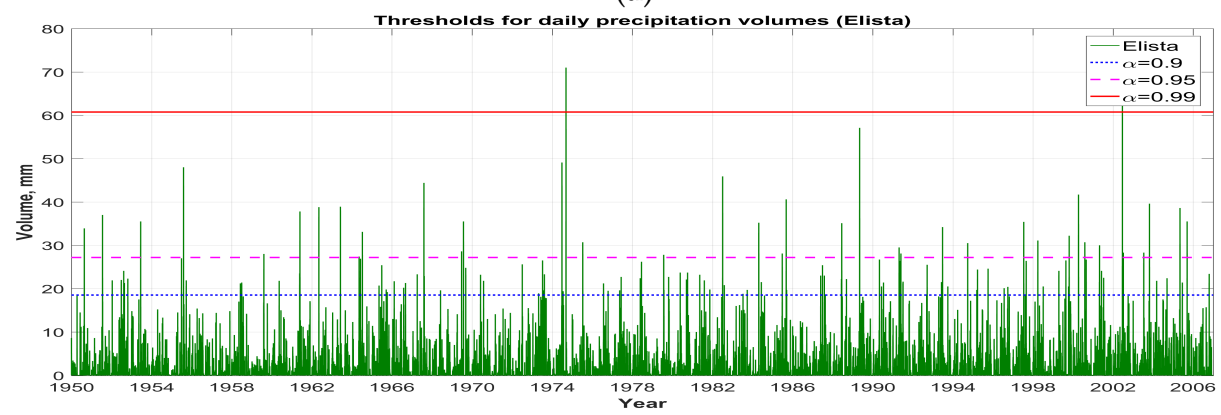

(b)

Figure 6. Testing daily precipitation within a wet period for abnormal heaviness: (a) Potsdam; (b) Elista, all data.

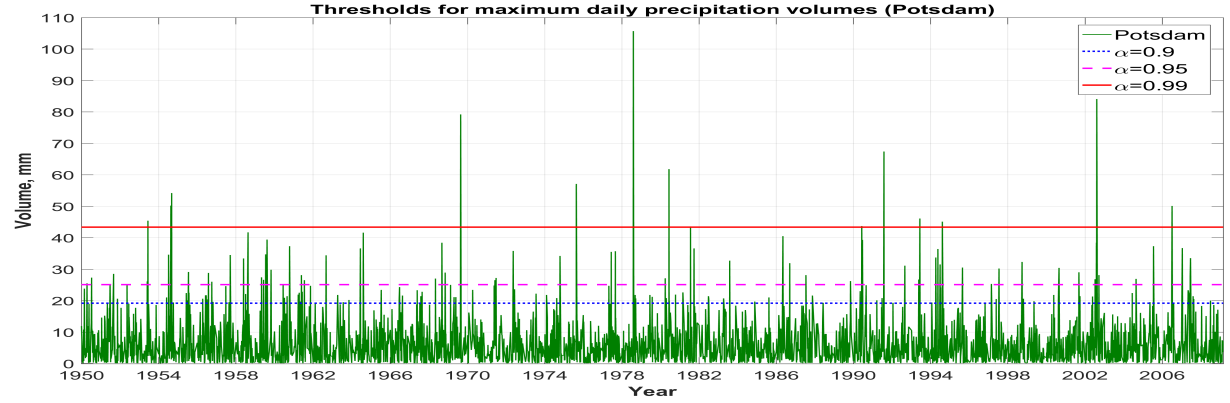

(a)

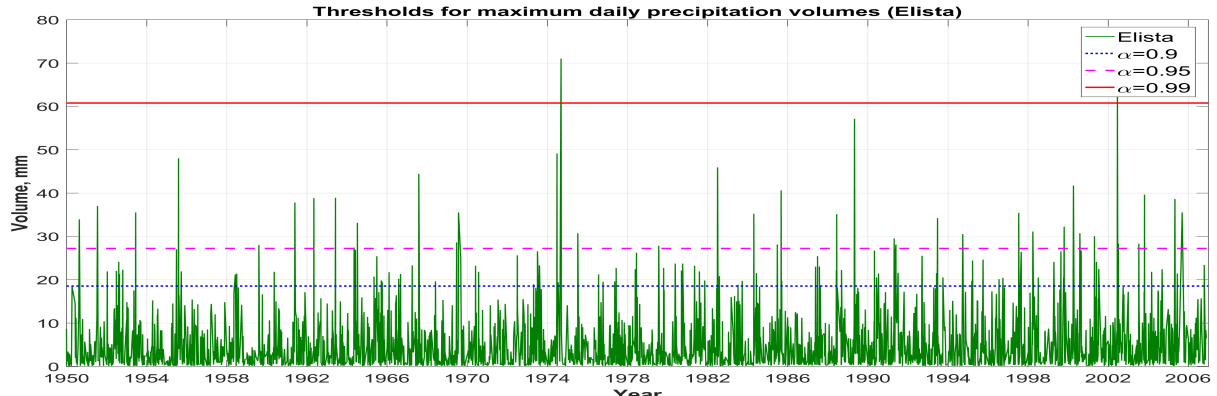

(b)

Figure 7. Testing maximum daily precipitation within a wet period for abnormal heaviness: (a) Potsdam; (b) Elista, data containing only maximum daily precipitation for every wet period.

During the same period in Elista there were only 2 wet periods containing anomalously heavy maximum daily precipitation volumes (at 99\% threshold) and 40 wet periods containing anomalously heavy maximum daily precipitation volumes (at 95\% threshold). Other maxima were 'regular.' The proportion of abnormal maxima exceeding 99\% and 95\% thresholds in Potsdam is quite adequate (the latter is approximately five times greater than the former) whereas in Elista this proportion 
is noticeably different. Perhaps, this can be explained by the fact that, for Elista, heavy rains are rare events.

\subsection{Comparison With the Extreme Precipitation Detected by the Beta-Distributed Tests}

At this section we present the results of comparison of the precipitation events from two samples both having the length 20 rains over the selected time-period (Elista and Potsdam). The extreme rain is detected as it passes the $95 \%$ quantile in the corresponding statistics. The first approach exploits the Beta distribution and it is based on the ratio between the selected precipitation and total precipitation sum over the sample. This test has been previously suggested in the paper [11]. It leads to the threshold $T H_{\text {beta }}$ where all necessary parameters of the Beta distribution have been estimated directly from the samples. The second threshold $T H_{S F}$ is determined using the tempered Snedecor-Fisher distribution (3) (see Section 2.1). The results of the comparison are presented in Table 3 where only two maximum rains are accounted. The column Decision indicates whether the corresponding volume is considered to be extreme according to the threshold values.

Table 3. An example of comparing the results with the previously proposed test.

\begin{tabular}{cccccc}
\hline City & Volume & Ratio & TH $_{\text {beta }}$ & $\mathbf{T H}_{\mathbf{S F}}$ & Decision \\
\hline \multirow{2}{*}{ Potsdam } & 28.7 & 0.45 & 0.25 & 28.66 & Yes/Yes \\
& 16 & 0.25 & 0.25 & 28.66 & Yes/No \\
\hline \multirow{2}{*}{ Elista } & 21.9 & 0.59 & 0.23 & 21.19 & Yes/Yes \\
& 5.2 & 0.14 & 0.23 & 21.19 & No/No \\
\hline
\end{tabular}

It is clearly seen that the Snedecor-Fisher test detects the extremal rains more accurately. Unlike the beta test, it selects only the rains that substantially extend the average or other heavy rains but which, however, are not extremely heavy. This means that the supposed scheme really can be applied to detect and forecast the potentially dangerous phenomena and to separate them from the substantial but not extremally big precipitations.

\subsection{Determination Of Abnormalities Types Based on the Results of the Statistical Analysis}

This section demonstrates the application of the statistical methodology based on the test (15) to determine extremeness of the precipitation volumes within wet periods. This method allows us to improve the quality of detecting events of such type in comparison with approaches based on thresholds.

It should be emphasized that the parameter $m$ of the Snedecor-Fisher distribution of the test statistic $S R_{0}(15)$ is tightly connected with the time horizon, the abnormality of precipitation within which is studied. Indeed, the average duration of a wet/dry period (or the average distance between the first days of successive wet periods) in Potsdam turns out to be $5.804 \approx 6$ days. So, one observation of a total precipitation during a wet period, on the average, corresponds to approximately 6 days. This means, that, for example, the value $m=5$ corresponds to approximately one month on the time axis, the value $m=15$ corresponds to approximately 3 months, the value $m=60$ corresponds to approximately one year. Figure 8 presents a flowchart illustrating the proposed algorithm.

It is important that the test for whether a total precipitation volume during one wet period is anomalously large can be applied to the observed time series in a moving mode. For this purpose, a window (a set of successive observations) should be determined. The number of observations in this set, say, $m$, is called the window width. The observations within a window constitute the sample to be analyzed. After the test has been performed for a given position of the window, the window moves rightward by one observation so that the leftmost observation at the previous position of the window is excluded from the sample and the observation next to the rightmost observation is added to the sample. The test is performed once more and so on. It is clear that each fixed observation falls in 
exactly $m$ successive windows (from $m$ th to $N-m+1$, where $N$ denotes the number of wet periods). Two cases are possible: (i) the fixed observation is recognized as anomalously large within each of $m$ windows containing this observation and (ii) the fixed observation is recognized as anomalously large within at least one of $m$ windows containing this observation. In the case (i) the observation will be called absolutely anomalously large with respect to a given time horizon (approximately equal to $m \cdot 5.804 \approx 6 m$ days). In the case (ii) the observation will be called relatively anomalously large with respect to a given time horizon. Of course, these definitions admit intermediate cases where the observation is recognized as anomalously large for $q \cdot m$ windows with $q \in\left[\frac{1}{m}, 1\right]$.
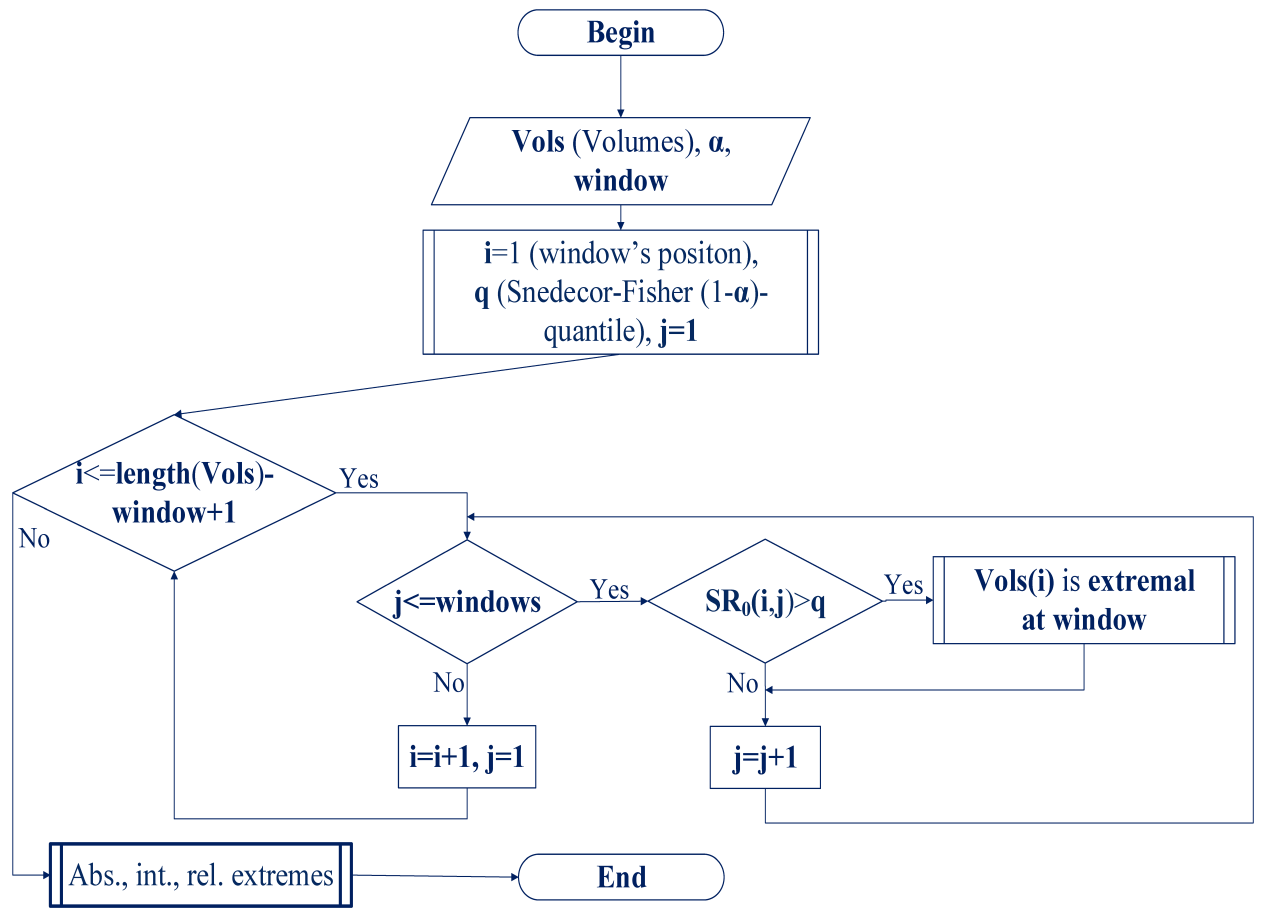

Figure 8. The algorithm of determination of extremeness of the precipitation volumes.

The results of the application of the test for a total precipitation volume during one wet period to be anomalously large based on $S R_{0}$ in the moving mode are shown on Figures 9-11 (Potsdam) and Figures 12-14 (Elista) for different time horizons (30, 90 and 360 days). The notation Extr int corresponds to the intermediate extremes (the fixed observation is recognized as anomalously large within at least $\lceil m / 2\rceil$ windows containing this observation, here the symbol $\lceil\cdot\rceil$ denotes the next larger integer). They are marked by circles on the figures. The absolutely extreme volumes are shown as triangles, and the relatively ones are marked by squares.

It is seen that at relatively small time horizons, the test yields non-trivial and unobvious conclusions. However, as the time horizon increases, the results of the test become more expected. At small time horizons there are some big precipitation volumes that are not recognized as abnormal. At large time horizons there are almost no regular big precipitation volumes at significance level $\alpha=0.05$ whereas at the smaller significance level $\alpha=0.01$ there are some regular big precipitation volumes which are thus not recognized as abnormal. 


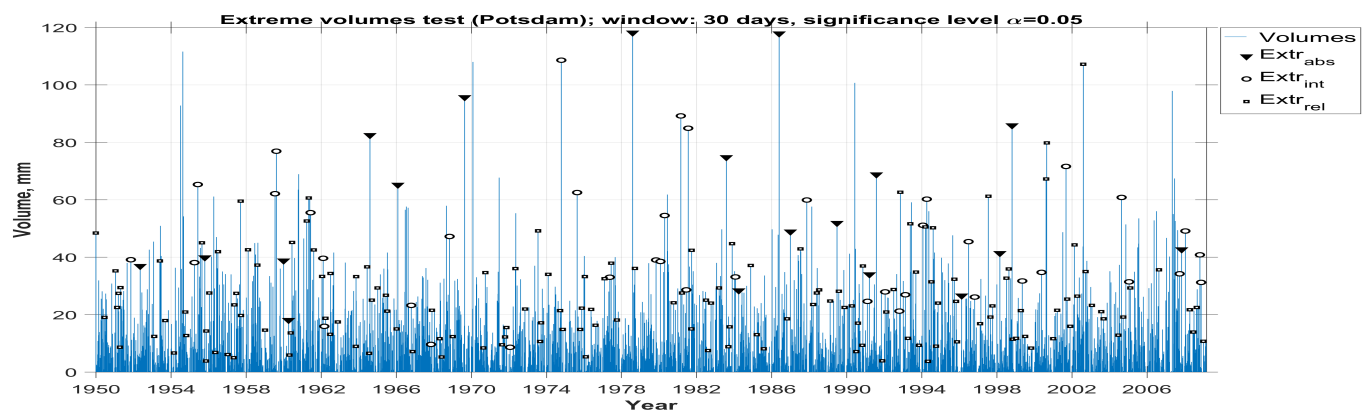

(a)

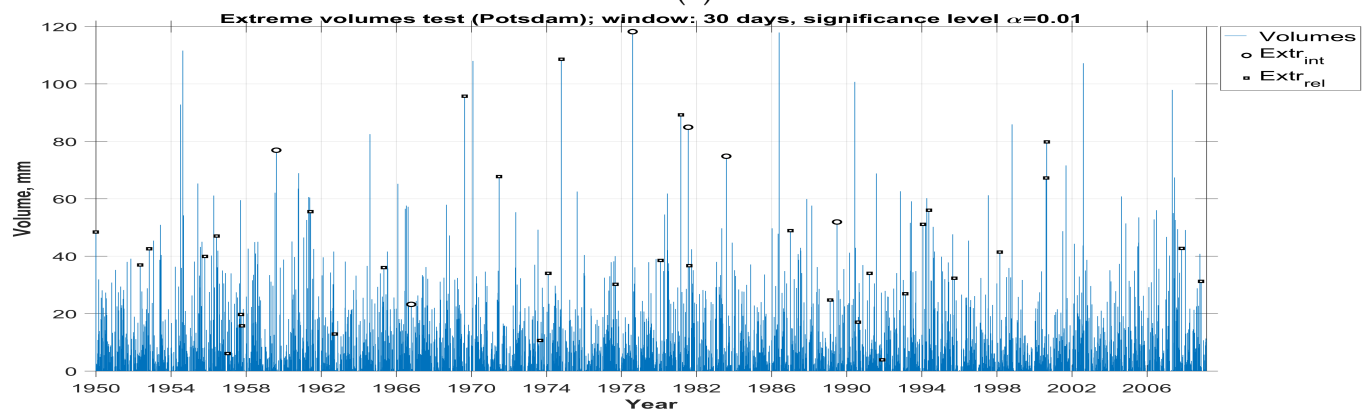

(b)

Figure 9. Abnormal precipitation volumes, Potsdam, time horizon $=30$ days, significance levels $\alpha=0.05$ (a) and $\alpha=0.01(\mathbf{b})$.

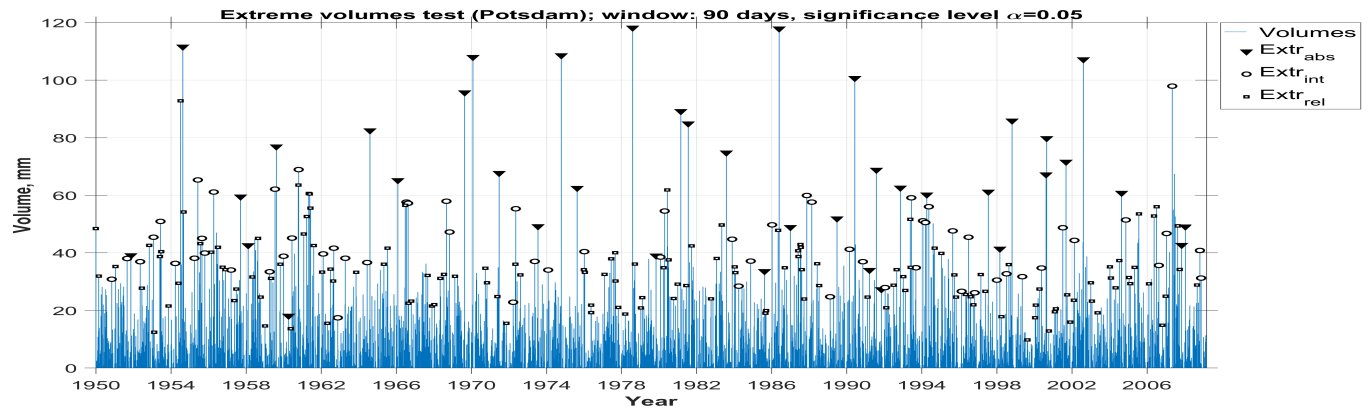

(a)

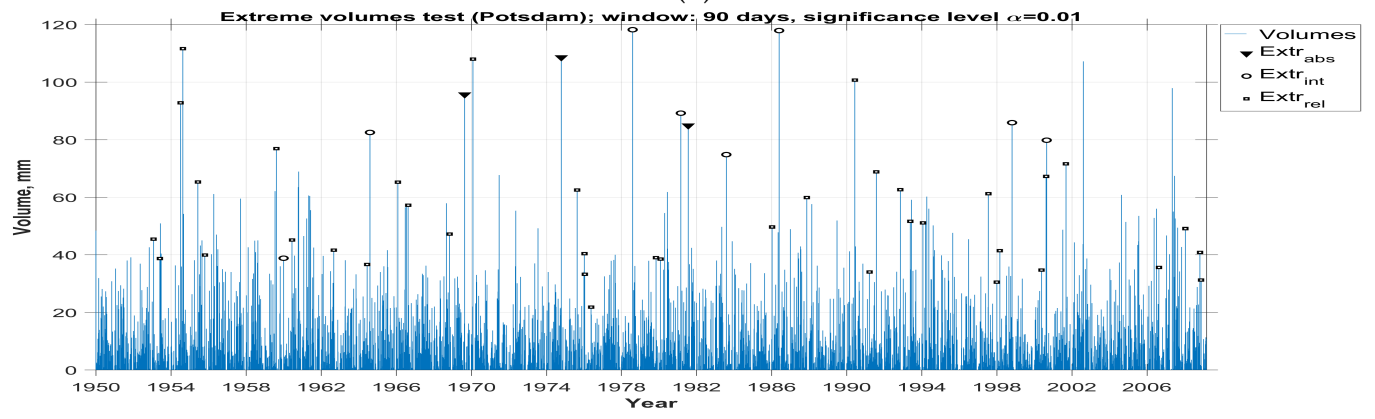

(b)

Figure 10. Abnormal precipitation volumes, Potsdam, time horizon $=90$ days, significance levels $\alpha=0.05$ (a) and $\alpha=0.01(\mathbf{b})$. 


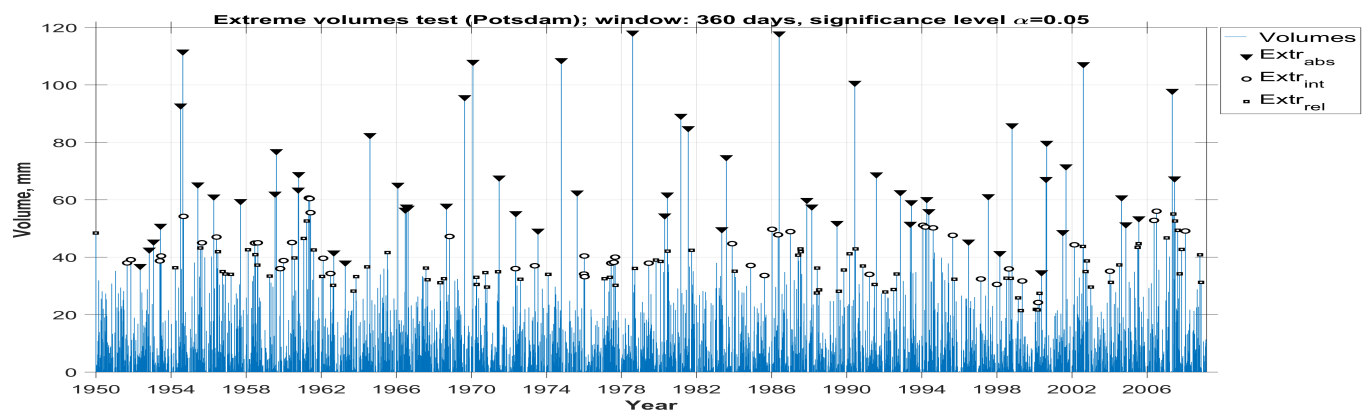

(a)

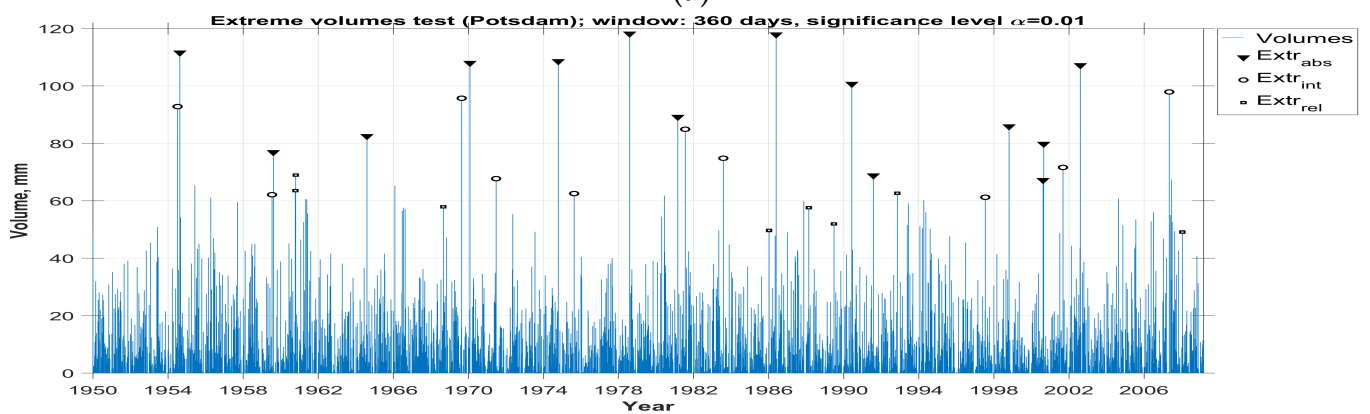

(b)

Figure 11. Abnormal precipitation volumes, Potsdam, time horizon $=360$ days, significance levels $\alpha=0.05$ (a) and $\alpha=0.01(\mathbf{b})$.

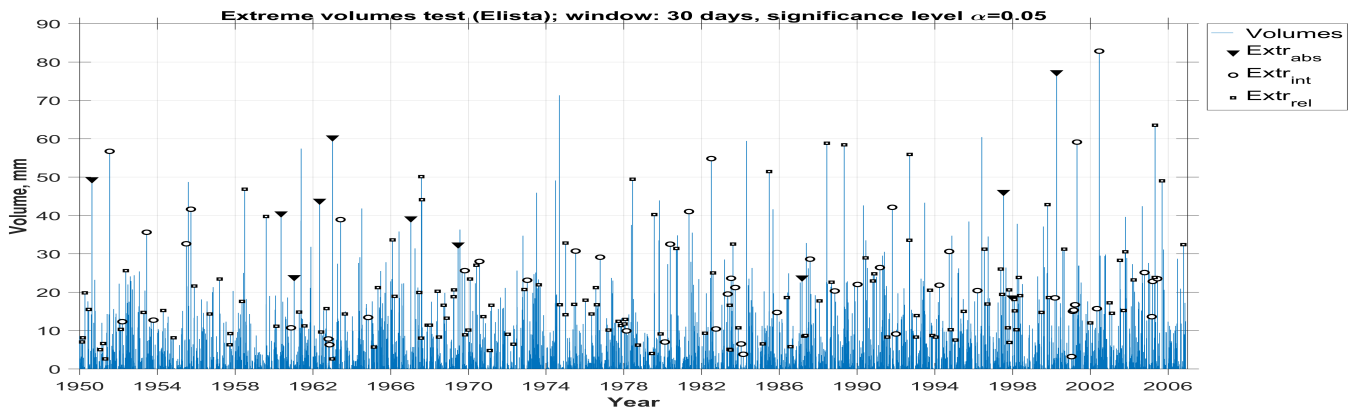

(a)

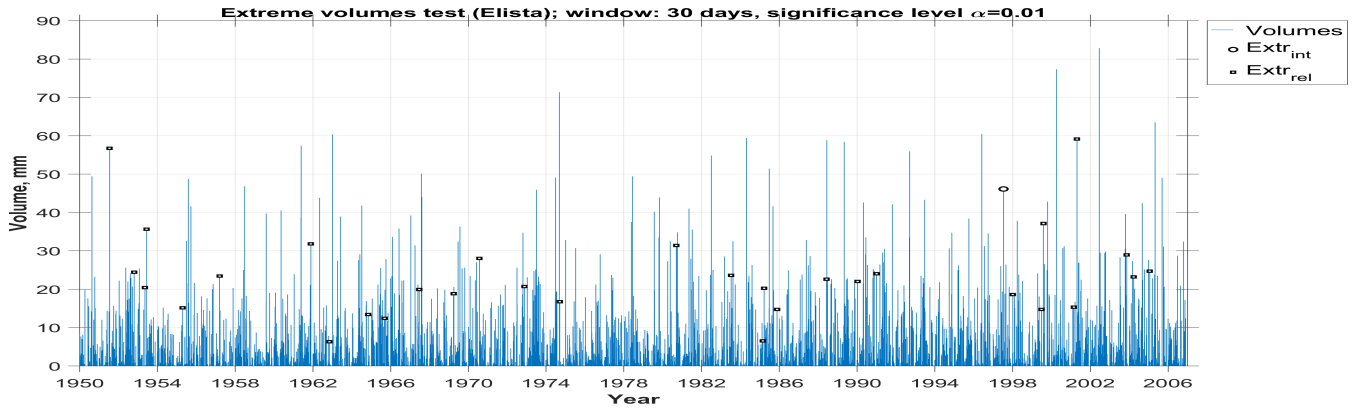

(b)

Figure 12. Abnormal precipitation volumes, Elista, time horizon $=30$ days, significance levels $\alpha=0.05$ (a) and $\alpha=0.01(\mathbf{b})$. 


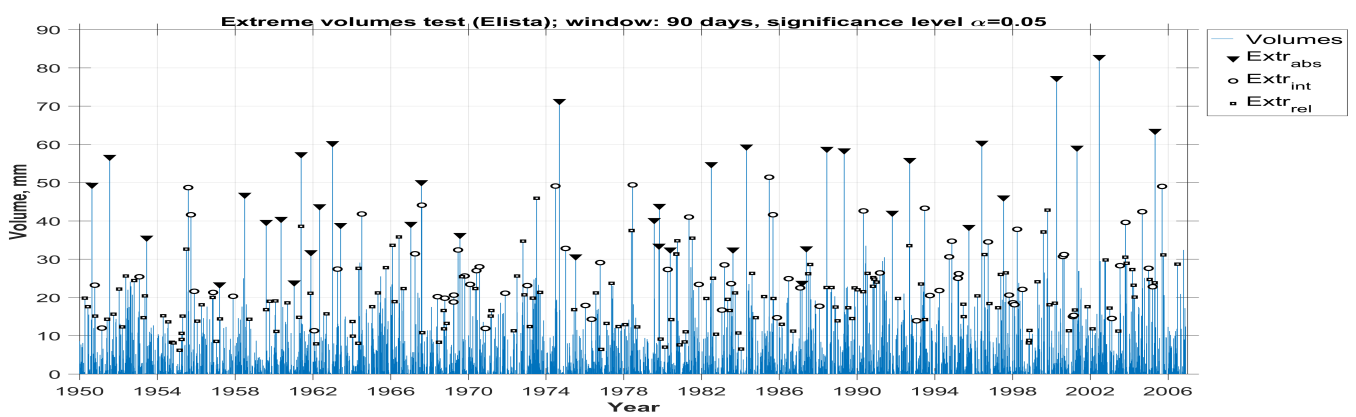

(a)

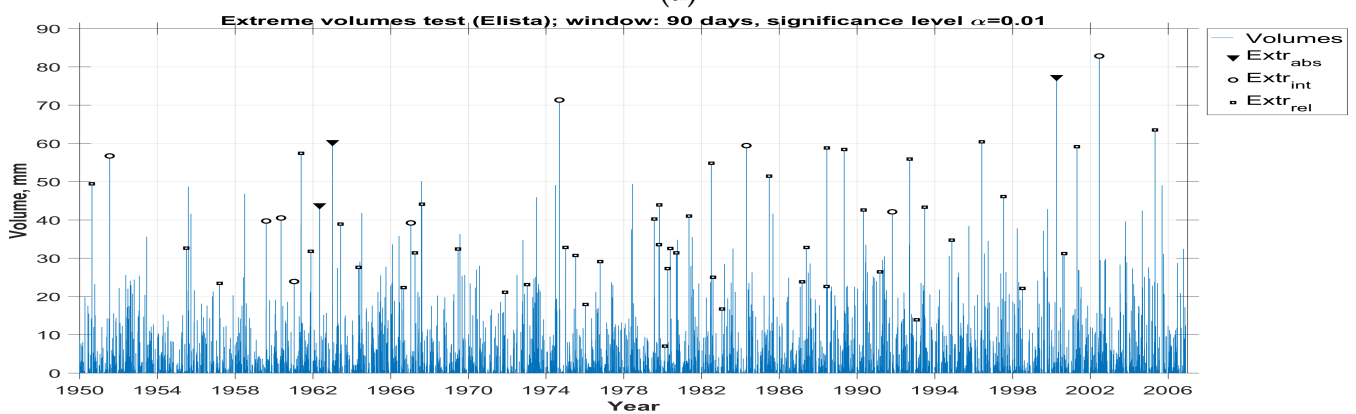

(b)

Figure 13. Abnormal precipitation volumes, Elista, time horizon $=90$ days, significance levels $\alpha=0.05$ (a) and $\alpha=0.01$ (b).

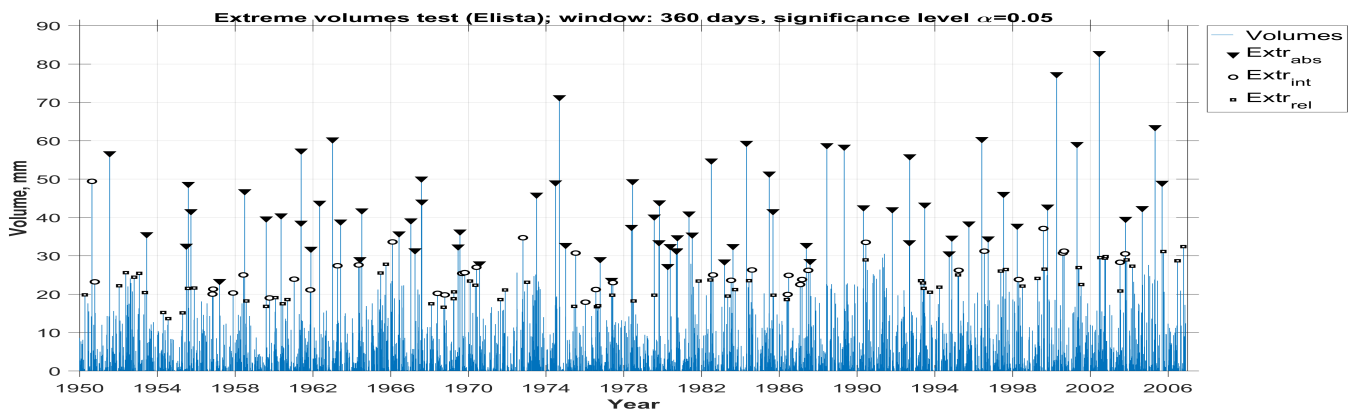

(a)

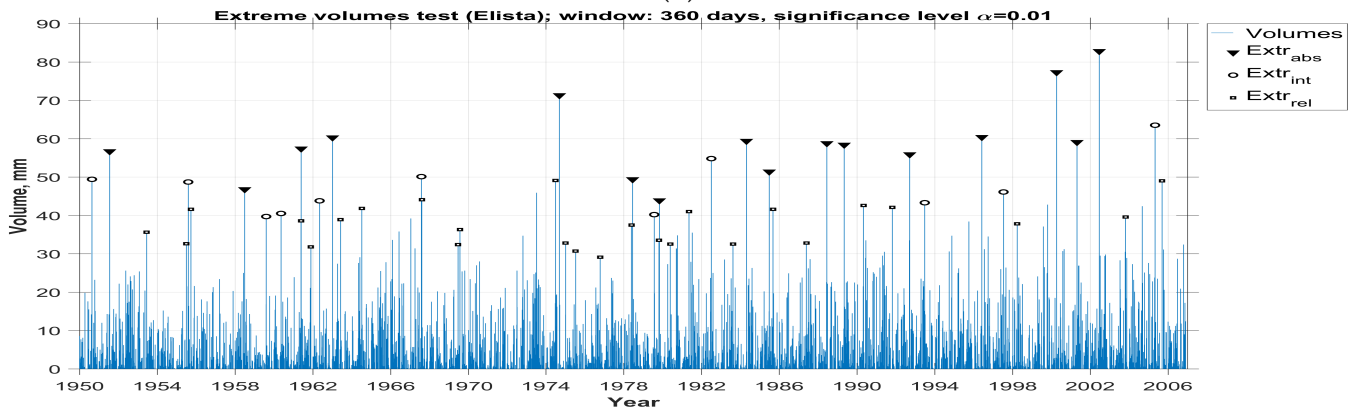

(b)

Figure 14. Abnormal precipitation volumes, Elista, time horizon $=360$ days, significance levels $\alpha=0.05(\mathbf{a})$ and $\alpha=0.01(\mathbf{b})$.

\section{Conclusions and Discussion}

In this paper, it is shown that the negative binomial distribution can be fruitful for the description of the statistical regularities in the duration of wet periods observed in practice and can be used as a base for obtaining the model for the extreme precipitation per wet period and, consequently, for testing the hypothesis that the specific precipitation volume considered over a given wet period is anomalously 
extreme. Several approaches to the definition of an anomalously extreme precipitation are proposed. This is an important issue, since up until now there has not been a single-valued criterion for which precipitation volume should be regarded as anomalous and which is not. Obviously, one and the same volume can be regarded normal in a region where precipitations are quite frequent, for instance in tropical zones and at the time it can be considered absolutely anomalous in a dry region, say, in a desert. The proposed tests consider the relative part of precipitation and the time scale and, therefore, are free from the aforementioned disadvantage. Moreover, the proposed techniques of testing the corresponding hypotheses can be easily numerically implemented.

The models and methods described in the paper can be applied to various time series in order to detect anomalously extreme values. For example, the considered algorithms can be used for other geophysical time series such as wind speed, heat fluxes and so forth, both univariate or multivariate. This can be important for global climate prediction models, for forecasting and evaluation of dangerous phenomena and processes. One more possible useful application of these models is filling the gaps in precipitation time series due to missing observations. Indeed, the statistically estimated parameters of the proposed models can be used as additional attributes in training samples when machine learning techniques or Artificial Intelligence systems are used for the simulation of missing data without actual extension of available information.

Author Contributions: Conceptualization, V.K., A.G., K.B.; Formal analysis, V.K., A.G.; Funding acquisition, A.G.; Investigation, V.K., A.G., K.B.; Methodology, V.K., A.G.; Project administration, V.K., A.G.; Resources, A.G., K.B.; Software, A.G.; Supervision, V.K.; Validation, A.G.; Visualization, A.G.; Writing-original draft, V.K., A.G.; Writing-review \& editing, V.K., A.G., K.B.

Funding: This research was funded by the Russian Foundation for Basic Research (grant number 17-07-00851) and the RF Presidential scholarship program (grant number 538.2018.5).

Acknowledgments: Authors thank the reviewers for their valuable comments that helped to improve the presentation of the material.

Conflicts of Interest: The authors declare no conflict of interest.

\section{References}

1. Groisman, P.; Legates, D. Documenting and detecting long-term precipitation trends: Where we are and what should be done. Clim. Chang. 1995, 31, 601-622. [CrossRef]

2. Groisman, P.; Karl, T.; Easterling, D.; Knight, R.; Jamason, P.; Hennessy, K.; Suppiah, R.; Page, C.; Wibig, J.; Fortuniak, K.; et al. Changes in the probability of heavy precipitation: Important indicators of climatic change. Clim. Chang. 1999, 42, 243-283.:1005432803188. [CrossRef]

3. Lockhoff, M.; Zolina, O.; Simmer, C.; Schulz, J. Evaluation of Satellite-Retrieved Extreme Precipitation over Europe using Gauge Observations. J. Clim. 2014, 27, 607-623. [CrossRef]

4. Zolina, O.; Simmer, C.; Belyaev, K.; Kapala, A.; Gulev, S.; Koltermann, P. Multidecadal trends in the duration of wet spells and associated intensity of precipitation as revealed by a very dense observational German network. Environ. Res. Lett. 2014, 9. [CrossRef]

5. Zolina, O.; Simmer, C.; Belyaev, K.; Kapala, A.; Gulev, S.; Koltermann, P. Changes in the duration of European wet and dry spells during the last 60 years. J. Clim. 2013, 26, 2022-2047. [CrossRef]

6. Mo, C.; Ruan, Y.; He, J.; Jin, J.; Liu, P.; Sun, G. Frequency analysis of precipitation extremes under climate change. Int. J. Climatol. 2019, 39, 1373-1387. [CrossRef]

7. Donat, M.; Angelil, O.; Ukkola, A. Intensification of precipitation extremes in the world's humid and water-limited regions. Environ. Res. Lett. 2019, 14. [CrossRef]

8. Bezak, N.; Auflic, M.J.; Mikos, M. Application of hydrological modelling for temporal prediction of rainfall-induced shallow landslides. Landslides 2019, 16, 1273-1283. [CrossRef]

9. Huang, J.; van Asch, T.; Wang, C.; Li, Q. Study on the combined threshold for gully-type debris flow early warning. Nat. Hazards Earth Syst. Sci. 2019, 19, 41-51. [CrossRef]

10. Bliznak, V.; Kaspar, M.; Muller, M.; Zacharov, P. Sub-daily temporal reconstruction of extreme precipitation events using NWP model simulations. Atmos. Res. 2019, 224, 65-80. [CrossRef] 
11. Zolina, O.; Simmer, C.; Belyaev, K.; Kapala, A.; Gulev, S. Improving estimates of heavy and extreme precipitation using daily records from European rain gauges. J. Hydrometeorol. 2009, 10, 701-716. [CrossRef]

12. Balkema, A.; de Haan, L. Residual life time at great age. Ann. Probab. 1974, 2, 792-804. [CrossRef]

13. Pickands, J. Statistical inference using extreme order statistics. Ann. Stat. 1975, 3, 119-131. [CrossRef]

14. Begueria, S.; Vicente-Serrano, S. Mapping the hazard of extreme rainfall by peaks over threshold extreme value analysis and spatial regression techniques. J. Appl. Meteorol. Climatol. 2006, 45, 108-124. [CrossRef]

15. Kysely, J.; Picek, J.; Beranova, R. Estimating extremes in climate change simulations using the peaks-over-threshold method with a non-stationary threshold. Glob. Planet. Chang. 2010, 72, 55-68. [CrossRef]

16. Begueria, S.; Angulo-Martinez, M.; Vicente-Serrano, S.; Lopez-Moreno, I.; El-Kenawy, A. Assessing trends in extreme precipitation events intensity and magnitude using non-stationary peaks-over-threshold analysis: A case study in northeast Spain from 1930 to 2006. Int. J. Climatol. 2011, 31, 2102-2114. [CrossRef]

17. Gorshenin, A.; Korolev, V. Determining the extremes of precipitation volumes based on a modified "Peaks over Threshold". Inform. Primenen. 2018, 12, 16-24. [CrossRef]

18. Huang, W.; Nychka, D.; Zhang, H. Estimating precipitation extremes using the log-histospline. Environmetrics 2019, 30. [CrossRef]

19. Gorshenin, A. Pattern-based analysis of probabilistic and statistical characteristics of precipitations. Inform. Primenen. 2017, 11, 38-46. [CrossRef]

20. Gorshenin, A. On some mathematical and programming methods for construction of structural models of information flows. Inform. Primenen. 2017, 11, 58-68. [CrossRef]

21. Korolev, V.; Gorshenin, A.; Gulev, S.; Belyaev, K.; Grusho, A. Statistical Analysis of Precipitation Events. AIP Conf. Proc. 2017, 1863. [CrossRef]

22. Vasilieva, M.; Gorshenin, A.; Korolev, V. Statistical analysis of probability characteristics of precipitation in different geographical regions. Adv. Intell. Syst. Comput. 2020, 902, 629-639._5. [CrossRef]

23. Zolotarev, V. One-Dimensional Stable Distributions; American Mathematical Society: Providence, RI, USA, 1986.

24. Kotz, S.; Ostrovskii, I. A mixture representation of the Linnik distribution. Stat. Probab. Lett. 1996, 26, 61-64. [CrossRef]

25. Korolev, V.; Gorshenin, A. The probability distribution of extreme precipitation. Dokl. Earth Sci. 2017, 477, 1461-1466. [CrossRef]

26. Gorshenin, A.; Korolev, V. Scale mixtures of Frechet distributions as asymptotic approximations of extreme precipitation. J. Math. Sci. 2018, 234, 886-903. [CrossRef]

27. Korolev, V. Convergence of random sequences with independent random indexes. I. Theory Probab. Appl. 1994, 39, 313-333. [CrossRef]

28. Korolev, V. Convergence of random sequences with independent random indexes. II. Theory Probab. Appl. 1995, 40, 770-772. [CrossRef]

29. Johnson, N.; Kotz, S.; Balakrishnan, N. Continuous Univariate Distributions, 2nd ed.; Wiley: New York, NY, USA, 1995; Volume 2.

30. Embrechts, P.; Klüppelberg, K.; Mikosch, T. Modeling Extremal Events; Springer: Berlin, Germany, 1998.

(C) 2019 by the authors. Licensee MDPI, Basel, Switzerland. This article is an open access article distributed under the terms and conditions of the Creative Commons Attribution (CC BY) license (http://creativecommons.org/licenses/by/4.0/). 